\title{
Selective Effects of Sport Expertise on the Stages of Mental Rotation Tasks With Object-Based and Egocentric Transformations
}

\author{
${ }^{1}$ School of Kinesiology, Shanghai University of Sport, Shanghai, China \\ ${ }^{2}$ China Institute of Sport Science, Beijing, China \\ ${ }^{3}$ Physical Education College of Zhengzhou University, Zhengzhou, China
}

Tian Feng ${ }^{1}$, Zhongqiu Zhang ${ }^{2}$, Zhiguang Ji', Binbin Jia ${ }^{1}$, and Yawei Li ${ }^{3}$

ABSTRACT

It is well established that motor expertise is linked to superior mental rotation ability, but few studies have attempted to explain the factors that influence the stages of mental rotation in sport experts. Some authors have argued that athletes are faster in the perceptual and decision stages but not in the rotation stages of object-based transformations; however, stimuli related to sport have not been used to test mental rotation with egocentric transformations. Therefore, 24 adolescent elite divers and 23 adolescent nonathletes completed mental rotation tasks with object-based and egocentric transformations. The results showed faster reaction times (RTs) for the motor experts in tasks with both types of transformations (object-based cube, object-based body, and egocentric body). Additionally, the differences in favour of motor experts in the perceptual and decision stages were confirmed. Interestingly, motor experts also outperformed nonathletes in the rotation stages in the egocentric transformations. These findings are discussed against the background of the effects of sport expertise on mental rotation.

\section{INTRODUCTION}

Mental rotation, defined as the ability to mentally manipulate two- or three-dimensional objects that may be rotated in any direction or translated in space (Shepard \& Metzler, 1971), is considered an indispensable component of spatial cognition. In the context of sports, a recent meta-analysis revealed an overall advantage in favour of sport experts in spatial tasks such as mental rotation (Voyer \& Jansen, 2017). For example, a football player should know his orientation precisely after turning around a certain angle, and it is extremely important for a diver who is learning a new movement to rehearse different skills and connect them in his or her mind. Therefore, the ability to manipulate actions mentally, which appears to play an essential role in the motor skills of athletes, has been found to be a reliable predictor of motor performance (Hoyek, Champely, Collet, Fargier, \& Guillot, 2014) and has thus received extensive attention. Additionally, the viewpoint of $\mathrm{em}$ - bodied cognition has been underlined by numerous studies concerning mental rotation in sport experts. This concept suggests that the cognitive ability of an individual is influenced by his or her environmental perception, and thus, cognitive processes are deeply rooted in the interactions between the body and the environment (Wilson, 2002). Hence, the relationship between mental rotation and sport environment is emphasized, and some essential findings have been identified.

First, the embodied nature of mental rotation tasks has been revealed. A number of studies have demonstrated that athletes in different sports, such as wrestling, gymnastics, orienteering, football, handball, fencing, and judoka, performed better on mental rotation

Corresponding author: Tian Feng, School of Kinesiology, Shanghai University of Sport, Shanghai, China. E-mail: teeeen@126.com 
than runners (Schmidt, Egger, Kieliger, Rubeli, \& Schüler, 2015) and nonathletes (Jansen \& Lehmann, 2013; Moreau, Clerc, Mansy-Dannay, \& Guerrien, 2011; Ozel, Larue, \& Molinaro, 2002, 2004; Pietsch \& Jansen, 2012; Schmidt et al., 2015). Researchers have clarified that in these activities, with more spatial reasoning demands, the mental representation of the body is continuously updated with regard to its position or movement. Athletes are asked to manipulate complex mental representations, such that mental rotation abilities could be embodied and benefit the most from these types of activities (Ionta \& Blanke, 2009; Moreau, Clerc, Mansy-Dannay, \& Guerrien, 2012).

Second, in mental rotation tasks, two transformations are distinguished: object-based transformations and egocentric (perspective) transformations (Zacks, Ollinger, Sheridan, \& Tversky, 2002). As vital moderators of the effect of sport expertise on mental rotation (Voyer \& Jansen, 2017), the former require same-different judgements about pairs of pictures, whereas the latter require left-right judgements about single pictures. In detail, in object-based transformations, an object is rotated and the observer's point of view remains fixed, such as a flying football being observed by the goalkeeper. In egocentric transformations, an object stays fixed while the observer's point of view rotates in relation to the object or the environment, such as the visual angle of a gymnast who is launching from one bar to another during a series of spins and twists. By measuring the two transformations of mental rotation in athletes, it has been suggested that both transformations could be embodied by sport expertise, though some studies have argued they do not share the same mechanism (Schmidt et al., 2015). Studies have found that athletes performed better than nonathletes in object-based mental rotation tasks with embodied (e.g., hand and human body) and nonembodied (e.g., cube and letter) objects (Jansen \& Lehmann, 2013; Jansen, Lehmann, \& Van Doren, 2012; Moreau et al., 2011; Ozel et al., 2002). However, some studies have observed that athletes outperformed nonathletes only when carrying out a left-right (egocentric) mental rotation task (Steggemann, Engbert, \& Weigelt, 2011). In line with that finding, Kaltner, Riecke, and Jansen (2014) demonstrated that sport expertise facilitated performance exclusively for egocentric transformations due to the human body stimulus eliciting embodied spatial transformations.

Contrary to the above results, Jola and Mast (2005) tested elite dancers and nondancers with a mental body rotation task and found no distinction between the two groups in the egocentric task. The researchers explained that the test stimuli were presented in different rotation axes from those in sport movements and impeded athletes in showing facilitation from expertise. Additionally, Habacha, LejeunePoutrain, Margas, and Molinaro (2014) demonstrated that mental rotations of human body figures with an outstretched arm may cause some athletes difficulties. It was found that the higher the correlation between the mental rotation task and the sport situation, the more spatial transformations were embodied; thus, better performance of the sport experts was found, revealing a selective effect of motor expertise. However, previous studies have neglected to consider whole-body rotations as well as more complex and sport-specific concurrent movements in mental rotation tasks (Heinen, 2013). Thus, the present study used the images of body postures appearing in sport movements (e.g., twisting in diving) rather than normal movements (e.g., arm stretching) to provide a better understanding of the interplay between sport expertise and the egocentric transformation in mental rotation.

Apart from the types of mental rotation, the stages of mental rotation were subdivided into perceptual stages (perceptual processing, stimuli identification and discrimination, orientation identification), rotation stages (mental rotation, parity judgement) and decision stages (response selection, execution, Heil \& Rolke, 2002; Shepard \& Cooper, 1986). According to Wright, Thompson, Ganis, Newcombe, and Kosslyn (2008), the individual contributions of particular stages cannot be specified by overall performance. Thus, computerized testing is used to facilitate the componential analysis of reaction times (RTs) and RTs are decomposed to reflect two components: the slope and the intercept of the RTs function at every angular disparity. The slope represents the process of the rotation stages, and the intercept represents the processes of perceptual and decision stages (Jansen et al., 2012; Just \& Carpenter, 1985).

However, controversies remain in the research on mental rotation stages. One example concerns which stages of mental rotation are more influenced by sport expertise and whether this effect is the same in both types of transformations. First, with respect to objectbased transformations, reports have shown that although athletes showed expertise in the perceptual and decision stages, they did not outperform nonathletes in mental rotation speed (Jansen et al., 2012). However, other studies showed that sport expertise was associated with better performance in all stages (Ozel et al., 2002). Second, few studies have identified the stages of egocentric transformations in athletes, so the effect of sport expertise on the stages of mental rotation remains unclear. From the viewpoint of embodied cognition, physical movement and motor imagery share a common process (Wohlschläger \& Wohlschläger, 1998), and researchers have found that accelerating the speed of motor rotation can correspondingly increase the mental rotation speed (Wexler, Kosslyn, \& Berthoz, 1998). Thus, if a person has experience in accelerating the body rotation speed, she or he may be able to perform better mental rotation speed than another person who does not have that experience.

To understand the contradictory findings in the types and stages of mental rotation, sports that involve rotational information should be considered. Diving (e.g., platform diving or springboard diving) is an event that includes abundant rotation skills (e.g., twists and somersaults) and different body axes (head-feet, front-back, and left-right) are employed by divers to optimize their performance. Though diving seems similar to other sports with spatial reasoning demands, such as gymnastics or trampoline, which have been investigated by some studies (Ozel et al., 2002), there are good reasons to pay attention to the mental rotation ability of divers. Compared with gymnastics, which allows the athlete to land on his or her feet, diving requires the athlete to rotate half somersault to enter the water with his or her head down, showing much greater skill requirements. Essentially, spatial perception is regarded as a basic competency for divers. For example, expert divers are able to correct their actions through nimbly recognizing 
their orientation and distance when they practice complex skills. Liang (2014) investigated spatial perception in divers on a national team and asked divers and nonathletes to judge the spatial position of an athlete at a certain time point during a dive. The results demonstrated that divers outperformed nondivers in terms of accuracy. Moreover, motor imagery is crucial for divers because they need to visualize diving movement accurately during training and also before competitions begin (Reed, 2002).

In addition, in assessments of mental rotation ability, little attention has been paid to adolescent participants. For example, a recent meta-analysis found participants of all available studies were older than 17 years of age (Voyer \& Jansen, 2017). The lack of children and adolescent subjects may be explained by the fact that expertise is developed over at least ten years of practice or with deliberate practice that begins in childhood (Ericsson, Krampe, \& Tesch-Römer, 1993). However, previous studies have demonstrated better mental rotation ability among children who regularly participate in sport training than those who do not. Jansen, Lange, and Heil (2011) assessed the mental rotation ability of two groups of girls aged 9-14 years. One group participated in juggling training for three months, and another group did light strength training. The results showed the experimental group performed significantly faster than the controls. Other studies have found a positive relationship between motor ability and accuracy in mental rotation tasks among primary school-aged and younger children (Jansen \& Heil, 2010; Jansen \& Kellner, 2015), indicating that motor ability could influence the overlap between motor and visual cognitive processes in children.

Therefore, the present study aimed to use nonembodied (cube) and embodied (sport-specific movement) stimuli to investigate the mental rotation abilities of adolescent elite divers and nonathletes with both object-based and egocentric transformations and to characterize the stages of the mental rotation process. Due to the abundant demands for the spatial reasoning and rotation skills of divers, it was predicted that the divers would perform better in both the object-based and egocentric mental rotation tasks (Hypothesis 1). Additionally, it was predicted that the divers would be faster in the perceptual and decision stages of object-based and egocentric tasks (Hypothesis 2). Divers are accustomed to imagining movements in a short time period before execution and they can accelerate the imagery period of movements when facing time constraints. Additionally, outstanding divers can voluntarily accelerate their rotation speed when jumping is delayed or they do not reach high enough. Thus, advantages in the rotation stages in favour of the divers were expected to be observed in egocentric tasks (Hypothesis 3).

\section{METHODS}

\section{Participants}

Forty-seven participants, including 24 athletes (11 males) aged 12-16 years $\left(M_{\text {age }}=14.41 ; S D=2.13\right)$ and 23 nonathletes (11 males) aged 1315 years $\left(M_{\text {age }}=13.91 ; S D=0.53\right)$, participated in the experiment. The group of athletes consisted of divers on the Shanghai diving team. The athletes' training age was between 8 and 13 years, and they had about 30 hours of diving practice per week. The group of nonathletes consisted of middle school students who had never taken part in competitive sport training. The two groups did not vary in terms of their ages, $F(1,42)=1.142, p=.291, \eta_{\mathrm{p}}^{2}=.026$. Informed consent was obtained prior to the experiment.

\section{Apparatus and Stimuli}

The two transformations were divided into three stimulus conditions in the present study. In the objected-based transformations, two conditions were presented. The objected-based cube (OC) condition presented two images of the cube simultaneously. The left image was upright as the target, and the right image was identical or mirrorreversed to the target but was presented at various angular disparities (see Figure 1). The angular disparities were $0^{\circ}, 30^{\circ}, 60^{\circ}, 90^{\circ}, 120^{\circ}, 150^{\circ}$, or $180^{\circ}$, and the stimuli were rotated with picture-plane rotations in a clockwise direction. In the objected-based body (OB) condition, two images of the body were presented. One image was the target and the other was a rotated identical or mirror-reversed image to the target. The body image was the back view of a female wearing a swimsuit with one arm bent over her head and another arm placed on her waist (see Figure 1). In the egocentric body (EB) condition, one image of the back view of a female was presented at a series of angular disparities (see Figure 1). Every image was shown as $4 \mathrm{~cm} \times 4 \mathrm{~cm}$ on a black screen. The stimuli appeared in black and white. The luminosity of the dark grey (colour of the dark grey cubes and the swimsuits) was $30 \mathrm{~cd} / \mathrm{m}^{2}$,

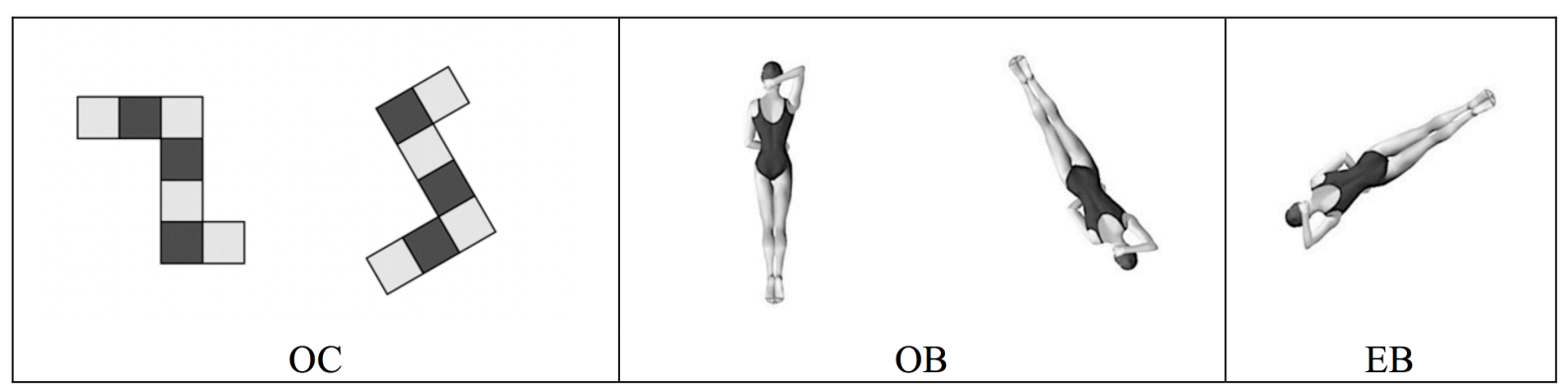

FIGURE 1.

Examples of three stimuli conditions (OC - objected-based cube, OB - objected-based body, EB - egocentric body). $\odot$ QA International, 2017. All rights reserved. 
the luminosity of the light grey (colour of the light grey cubes and the arms and legs of the humans) was $80 \mathrm{~cd} / \mathrm{m}^{2}$, and the luminosity of the white background was $100 \mathrm{~cd} / \mathrm{m}^{2}$. The task was designed and displayed with E-Prime 2.0 (Psychology Software Tools, www.pstnet.com) on ThinkPad laptops with 14 in. screens.

\section{Procedure}

The experiment was conducted in a quiet room at the Sport University of Shanghai, and each participant was tested individually under the supervision of an experimenter. First, the participants completed a questionnaire with individual information and then were seated in front of the screen at a distance of $60 \mathrm{~cm}$. After reading standardized task instructions, subjects were asked to practice 20 trials with feedback. The error rate (ER) had to be below $20 \%$ to ensure that they had completely understood the task. All participants were required to correctly answer at least 16 of the 20 practice trials. Otherwise, they had to perform an additional 20 practice trials to reach the requirement. In the $\mathrm{OB}$ and $\mathrm{OC}$ conditions, participants needed to determine as quickly and as accurately as possible whether the two images were the same, regardless of the angular disparity. In the EB condition, participants were asked to decide which arm the female had bent over her head. The experiment consisted of 3 (condition) $\times 7$ (angular disparity) $\times 2$ (left-right or same-different) $\times 4$ (repetition) trials for a total of 168 trials divided into three blocks. Blocks and trials were randomly ordered. In each trial, the screen showed a fixation ( $1 \mathrm{~s}$ to $1.5 \mathrm{~s}$ ), and then, the image/s was/were presented until the $F$ (for left/same) or $J$ (for right/different) key was pressed by the subject (no more than 3 s). Subsequently, a blank screen without feedback was presented for 1 $s$, and the succeeding trial started immediately. The entire experiment took approximately $25 \mathrm{~min}$.

\section{Statistical Analyses}

Before the analyses were conducted, RTs more than three SDs above the mean, participants with ERs above 25\% (two athletes and one nonathlete), and RTs for incorrect trials (6.9\%) were excluded. RTs of two groups in the three conditions were transformed into a logarithmic base (ln) and showed normal distributions $(z<1.097, p>.180$, in all instances). Moreover, normal distributions were presented for the ERs of two groups in each condition ( $z<1.345, p>.054$, in all instances).

To test Hypothesis 1, regarding whether divers and nonathletes differed in terms of their performance on various mental rotation tasks, two analyses of variance (ANOVAs) for the dependent variables RT and ER were calculated with the between-subjects factor of group (divers, nonathletes) and the within-subject factors of angular disparity $\left(0^{\circ}, 30^{\circ}, 60^{\circ}, 90^{\circ}, 120^{\circ}, 150^{\circ}\right.$, and $\left.180^{\circ}\right)$ and condition (OC, OB, and $\mathrm{EB})$. Bonferroni-corrected post hoc $t$ tests were used for identifying the main effects and interactions. Moreover, the RT functions of angular disparity were presented for each combination of condition and group. The slope of the linear component of the RT function represents the millisecond increment in RT, associated with a $1^{\circ}$ increment in orientation. Thus, the inverse of the slope multiplied by 1,000 shows the mental rotation speed, which is how many degrees could be rotated in one second. Moreover, the intercept represents the RT at the angular disparity of $0^{\circ}$, which is a good estimator of the components of task performance other than the rotation stages, including stimulus encoding and responding (Yu \& Zacks, 2015). Thereby, Hypotheses 2 and 3 concerning the perceptual and decision stages versus the rotation stages were tested, respectively. With respect to the perceptual and decision stages, three independent-samples $t$ tests for RT at $0^{\circ}$ in the three conditions between the athletes and the nonathletes were conducted separately. For the rotation stages, one ANOVA with repeated measures for the dependent variable of mental rotation speed was calculated with the between-subjects factor of group (divers, nonathletes) and the within-subject factor of condition (OC, OB, and EB). Moreover, three independent-samples $t$ tests were also performed separately for mental rotation speed in the three conditions to compare performance of the athletes with that of the nonathletes.

\section{RESULTS}

\section{Reaction Time}

The ANOVA revealed significant main effects of group, $F(1,42)=$ $10.339, p<.01, \eta_{\mathrm{p}}{ }^{2}=.198$, showing that the RTs of divers (OC: $M=$ $1,818, S D=396 ;$ OB: $M=1,469, S D=245 ; \mathrm{EB}: M=827, S D=175$ ) were faster than that of nonathletes (OC: $M=2,556, S D=931$; OB: $M$ $=1,995, S D=862$; EB: $M=1,136, S D=614$, see Figure 2). Additionally, significant main effects of angular disparity, $F(6,252)=179.974, p<$ $.001, \eta_{\mathrm{p}}{ }^{2}=.811$, and condition, $F(2,84)=371.282, p<.001, \eta_{\mathrm{p}}{ }^{2}=.898$, and their interaction, $F(12,504)=11.287, p<.001, \eta_{p}{ }^{2}=.212$, were also confirmed (see Figure 3). Post hoc tests of RTs in the three conditions for each angular disparity found that the $\mathrm{RT}$ in the $\mathrm{OB}$ condition was faster than that in the OC condition at every angular disparity $(t>$ $3.438, p<.001)$, except $150^{\circ}(t=1.534, p=.129)$ and $180^{\circ}(t=-0.542, p$ $=.589$ ), and the $\mathrm{RT}$ in the $\mathrm{EB}$ condition was faster than that in the $\mathrm{OC}$ condition and the $\mathrm{OB}$ condition at every angular disparity $(t>6.320$, all $p<.001$, in all instances). Nevertheless, there were no significant interactions between group and angular disparity, $F(6,252)=0.616, p$ $=.660, \eta_{\mathrm{p}}{ }^{2}=.014$, or group and condition, $F(2,84)=0.363, p=.660$,

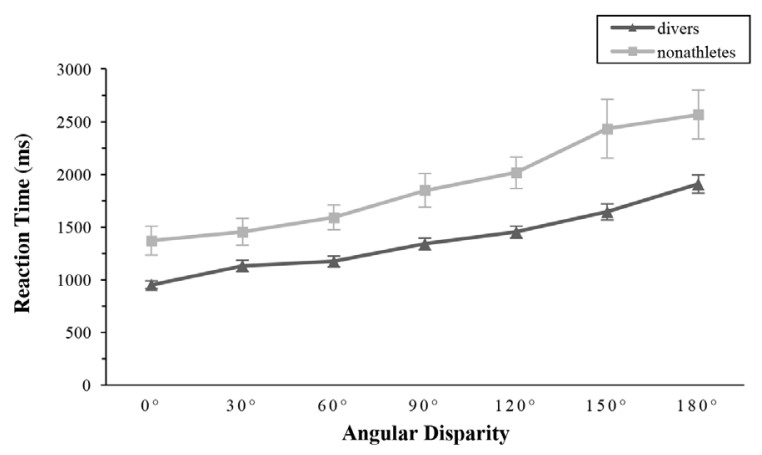

FIGURE 2.

Reaction time (mean and $S E$ ) averaged across conditions for each group at each angular disparity. 


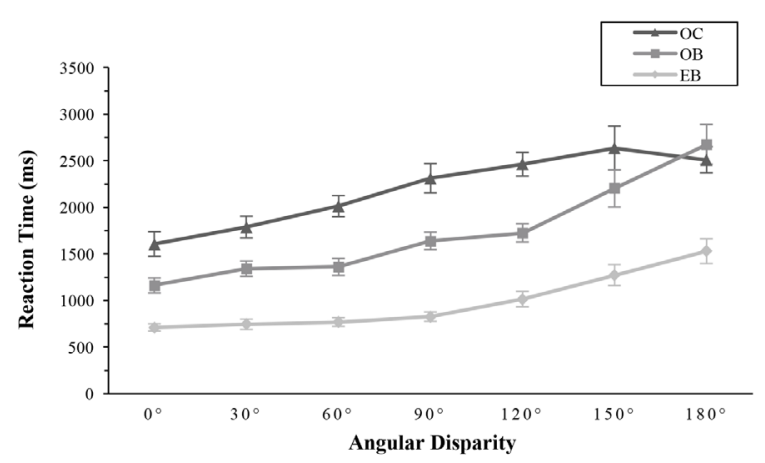

FIGURE 3.

Reaction time (mean and $S E$ ) averaged across groups for each condition at each angular disparity.

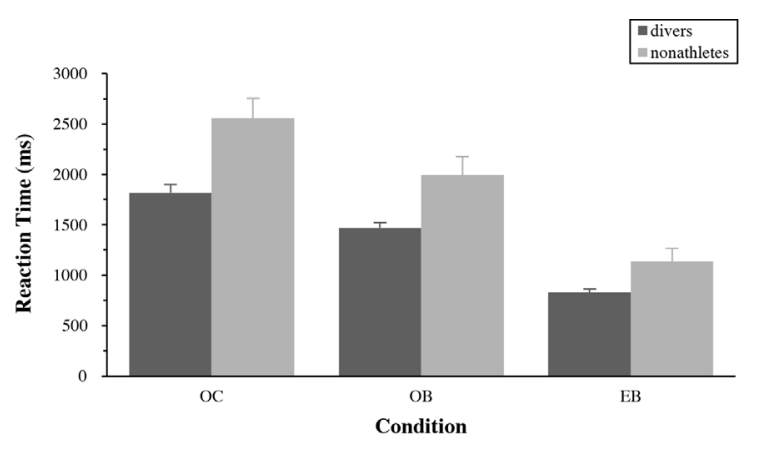

FIGURE 4.

Reaction time (mean and SE) for each group and each condition.

$\eta_{\mathrm{p}}{ }^{2}=.009$, indicating that at various angular disparities and stimulus types, the distinctions between groups in RTs remained significant (see Figure 4)

\section{Error Rate}

Similar analyses carried out on ER indicated significant main effects of angular disparity, $F(6,252)=17.594, p<.001, \eta_{\mathrm{p}}{ }^{2}=.295$, and condition, $F(2,84)=21.273, p<.001, \eta_{\mathrm{p}}{ }^{2}=.336$, but not group, $F(1,42)=0.083$, $p=.775, \eta_{\mathrm{p}}{ }^{2}=.002$, which meant that divers (OC: $M=.102, S D=.055$; OB: $M=.088, S D=.061$; EB: $M=.020, S D=.013)$ and nonathletes (OC: $M=.108, S D=.113$; OB: $M=.070, S D=.054$; EB: $M=.020, S D$ $=.022)$ did not differ with regard to ER. Additionally, the interaction between angular disparity and condition was significant, $F(12,504)=$ 2.342, $p<.05, \eta_{\mathrm{p}}{ }^{2}=.053$. Post hoc tests of ERs in the three conditions for each angular disparity found that $\mathrm{ER}$ in the $\mathrm{OB}$ condition was lower than that in the OC condition at only $180^{\circ}(t=2.788, p<.01)$ but not for other angular disparities ( $t<1.816, p>.073$, in all other instances), and the ER in the EB condition was lower than that in the OC condition and the $\mathrm{OB}$ condition at every angular disparity $(t>2.021, p<$ $.049)$.

\section{Mental Rotation Stages}

Regarding the perceptual and decision stages, three independent-samples $t$ tests indicated that the divers (OC: $M=1,314, S D=417$, OB: $M$
$=925, S D=182, \mathrm{~EB}: M=612, S D=104)$ outperformed the nonathletes (OC: $M=1,900, S D=1,114$, OB: $M=1,401, S D=653$, EB: $M=169$, $S D=77$ ) across all conditions (OC: $t[42]=2.354, p<.05, d=0.60$; OB: $t[42]=3.283, p<.01, d=0.99$; EB: $t[42]=2.736, p<.05, d=0.82$ ).

With respect to the rotation stages, the results of an ANOVA showed the main effects of group, $F(1,42)=8.737, p<.01, \eta_{\mathrm{p}}{ }^{2}=.172$, and condition, $F(2,84)=50.130, p<.001, \eta_{\mathrm{p}}{ }^{2}=.544$, but not the interaction of them, $F(2,84)=1.747, p=.190, \eta_{\mathrm{p}}{ }^{2}=.040$. Alternatively, three $t$ tests were conducted to see if the ANOVA neglected some different patterns in the rotation speed of the divers and non-athletes. Three independent-samples $t$ tests revealed a faster rotation speed of the athletes (OC: $M=81, S D=60$, OB: $M=95, S D=41, \mathrm{~EB}: M=236$, $S D=107$ ) than the nonathletes (OC: $M=54, S D=47$, OB: $M=80, S D$ $=51$, EB: $M=169, S D=77)$ only in the EB condition, $t(42)=2.389, p<$ $.05, d=0.72$, and not for the OC condition, $t(42)=1.539, p=.131, d=$ 0.46 , or $\mathrm{OB}$ condition, $t(42)=1.083, p=.285, d=0.33$. The results are shown in Figures 5 and 6.

\section{DISCUSSION}

The purposes of the present study were to investigate the effect of sport expertise on a mental rotation task with object-based and egocentric transformations and to clarify the characteristics of the stages of the mental rotation process. Performance of rotational sport experts

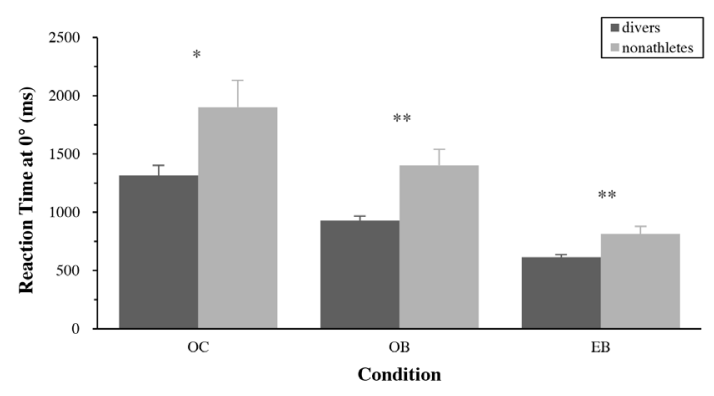

FIGURE 5.

Reaction time at $0^{\circ}$ (mean and $S E$ ) for each group and each condition.

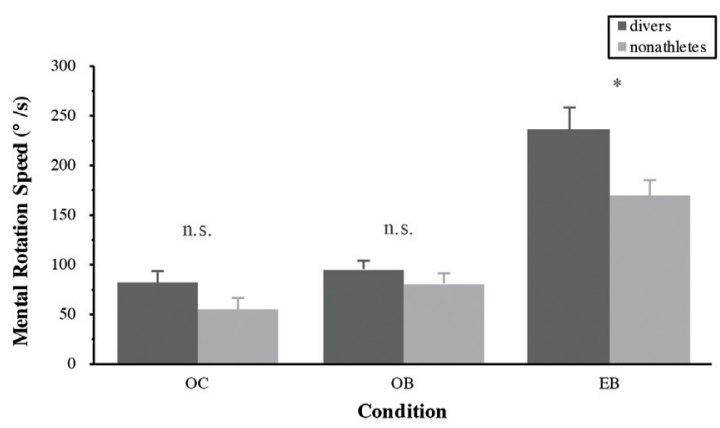

FIGURE 6.

Mental rotation speed (mean and SE) for each group and each condition. 
(elite divers) was compared to that of nonathletes in object-based and egocentric mental rotation tasks with cubes and sport-specific body figures. In the present study, athletes were adolescent elite divers with excellent performance ( 16 of 24 divers were in the top eight at national or international junior championships) and also started diving practice at very young ages (3-5 years old). This study is the first one which investigates mental rotation performance in real adolescent experts and thus, might add important information to the present literature (Voyer \& Jansen, 2017).

As most researchers have shown (Jola \& Mast, 2005; Shepard \& Metzler, 1971; Voyer \& Bryden, 1990), RTs increased gradually as a function of angular disparity, which means that the more the angle was rotated, the more time the participants required to react. This result supported the functional equivalence hypothesis, stating that humans' perception is functionally equivalent to the physical phenomena surrounding them (Heinen \& Jeraj, 2013). In addition, post hoc analysis of every angular disparity in the three conditions illustrated that both divers and nonathletes had slower RTs and more ERs in the OC and $\mathrm{OB}$ conditions than in the $\mathrm{EB}$ condition, replicating the results of Jansen and Lehmann (2013), Jola and Mast (2005), Kaltner et al. (2014), and Kaltner and Jansen (2015). Additionally, faster RTs in OB than in OC condition at some angular disparities and a lower ER in $\mathrm{OB}$ than in $\mathrm{OC}$ condition at $180^{\circ}$ were found. These findings are supported by the idea of the heterogeneity of transformations, which is illustrated by the viewpoint of embodied cognition. This viewpoint suggests that an individual's perception of the environment could affect his or her cognitive ability, and cognitive processes are deeply rooted in the interactions between the body and the environment (Wilson, 2002). Thus, in an egocentric judgement, the judgements of postures were accelerated because the participants adopted more body-related experience learned from the first-person perspective in daily life that can facilitate such learning. Additionally, the results were supported by Kaltner and Jansen (2015), who compared the participants with positive body awareness (elite athletes), participants with negative body awareness (patients with Anorexia Nervosa), and healthy participants as a control group. They found that both special-population groups had a faster RT than the control group, showing that the processes in the body influenced mental rotation performance.

As for Hypothesis 1, the analysis of RT showed an effect of sport expertise in favour of divers, independent of the transformations. Additionally, unlike the results of Steggemann et al. (2011), which showed that athletes had a faster RT but a higher ER, the ERs of the two groups in the present study did not significantly differ. First, our study corroborated the advantage of divers in the OC condition because the divers had a faster RT, independent of the angular disparities, and smaller standard deviations of RTs at higher angular disparities than the nonathletes. These findings confirmed that same-different judgements of cubes could also be embodied by sport experts, which was supported by the findings showing that experience with spatial activities could be transferred to higher cognitive abilities, such as mental rotation (Moreau, 2015). The results were consistent with some studies (Jansen \& Lehmann, 2013; Jansen et al., 2012). Nevertheless, other studies did not find such a distinction (Kaltner \& Jansen, 2015; Kaltner et al., 2014; Steggemann et al., 2011). In addition, the same advantage in favour of the divers was found in the OB condition, which supported Jansen et al. (2012) and Jansen and Lehmann (2013). Additionally, Amorim, Isableu, and Jarraya (2006) concluded that the body characteristics of the stimuli could enhance the extent of embodiment, even in a same-different judgement. In the EB condition, compared with subjects without sport experience, divers gained adequate information about body rotation, which could facilitate the embodied process in the mental rotation task. Consequently, it seemed that divers used the process of mapping their body axes onto the stimuli, which was similar to the motor process of imitating postures in sport (Amorim et al., 2006). This result was also supported by some studies (Kaltner \& Jansen, 2015; Kaltner et al., 2014; Steggemann et al., 2011). Kaltner et al. (2014) found that athletes could perform better in the mental rotation task due to their greater kinaesthetic representation and motor simulation abilities.

Additionally, our study demonstrated that the divers outperformed the nonathletes independent of the angular disparity in the egocentric transformation. However, Steggemann et al. (2011) investigated experts of rotational movements with an egocentric mental body rotation task and found that motor experts benefited only from the human figure in unfamiliar orientations. Their findings supported similar findings by Kaltner and Jansen (2015), who found that the two groups were significantly different only at the angular disparities of $135^{\circ}$ and $180^{\circ}$. More precisely, they suggested that the expertise-specific body representations gathered through kinaesthetic experiences were easily utilized in unusual body orientations. Regarding our result, it seemed that the stimulus of a diver's posture rather than a more general one could account for this independent effect; therefore, divers' kinaesthetic experience facilitated the process of mental rotation due to the conformity of sport movements and the characteristics of the tasks (Jola \& Mast, 2005). In line with that, Heppe, Kohler, Fleddermann, and Zentgraf (2016) assessed the mental rotation ability of volleyball and handball players in their Experiment 1 by asking them to discriminate between orientations of a human body but did not find any significant differences. Nevertheless, a human stimulus holding a ball in one hand was used in their Experiment 2, and a discrepancy between groups at a certain angular disparity was observed.

To determine the effect of sport expertise on the stages of mental rotation, we measured the $\mathrm{RT}$ at $0^{\circ}$ and the mental rotation speed of divers and nonathletes in the three conditions. First, the present study demonstrated that divers had faster perceptual and decision times in all three conditions, which corroborated Hypothesis 2. The result was supported by prior studies (Heppe et al., 2016; Jansen et al., 2012; Ozel et al., 2002, 2004). We used two-dimensional cubes (the OC condition) as stimuli, similar to Ozel et al. (2002), and replicated the differences between groups in the perceptual and decision stages. Jansen et al. (2012) revealed a better mental rotation $\mathrm{RT}$ at $0^{\circ}$ for soccer players than for nonathletes in an object-based body task (similar to the OB condition), and Heppe et al. (2016) determined that athletes performed better when responding to egocentric human figures (similar to the 
EB condition) at the $0^{\circ}$ position in their Experiment 2 . It is thought that the effect could be attributed to the encoding process, the maintenance of readiness, or the motor process in a mental rotation task. This explanation could also be strengthened by some studies (Heirani, Vazinitaher, Soori, \& Rahmani, 2012; Kioumourtzoglou, Kourtessis, Michalopoulou, \& Derri, 1998; Piras, Lobietti, \& Squatrito, 2014; Zwierko, Osinski, Lubinski, Czepita, \& Florkiewicz, 2010) that found that athletes had shorter simple RTs and choice RTs than nonathletes due to the effect of rapid, visually-demanding sports on the central nervous system. Taken together, our results show that divers can efficiently perceive when mentally rotating familiar motor postures as well as unfamiliar abstract cubes and make faster decisions.

Regarding the rotation stages, a majority of studies failed to reveal any differences between groups (Heppe et al., 2016; Jansen et al., 2012), so it seemed motor experience may not benefit the rotation stages in mental rotation. Interestingly, when the stimulus was substituted for a diving posture with a left-right judgement, divers could rotate faster than nonathletes, providing support for Hypothesis 3 . This finding was strengthened by Wexler et al. (1998), demonstrating the correlation of the rotation speed in mental rotation and motor rotation. Theoretically, the "body analogy" perspective proposes two kinds of embodiment during the performance of embodied spatial transformations: spatial embodiment and motoric embodiment (Amorim et al., 2006). The former means that the body axes can be mapped onto the embodied object, and the latter refers to the process of observing, imagining, or executing actions to the same motor representations (Decety, 2002; Vereza, 1999). In alignment with this perspective, the body axes were mapped to the objects (e.g., cube and body) by the divers. Thus, the encoding process was meditated, and a faster RT at $0^{\circ}$ of the divers was demonstrated. Concerning the rotation stages, given that the divers could covertly imitate the posture while mentally rotating (Heinen, 2013; Wexler et al., 1998), motoric embodiment helped them to maintain the postural spatial configuration during the mental rotation process. This facilitation may offer explanations for the faster mental rotation speed of divers (Amorim et al., 2006). Additionally, other factors, such as the transformation of the task, the stimulus material or the characteristics of participants in the experiment, may also influence mental rotation speed. Jansen et al. (2012) applied object-based transformation using stimuli of a nonsport body posture in that study, and athletes dealt with the task from a third person view so that they had little possibility to use motor-related body representations. In another study by Heppe et al. (2016), the researchers found that recreational athletes selected as the control group were also physically active, which could explain the lack of differences in mental rotation speed.

\section{CONCLUSION}

The present study considered the effect of sport expertise on mental rotation with object-based and egocentric transformations by differentiating the main stages of mental rotation. It was confirmed that divers had an advantage in mental rotation with both types of transforma- tions. More precisely, the study illustrated that experts performed faster than nonathletes in the perceptual and decision stages in both transformations and also demonstrated faster speeds in the rotation stages in the egocentric tasks. The results confirmed that same-different judgements of cubes could also be embodied by sport experts. The study also presented for the first time that embodied cognition could facilitate the speed of rotation stages in egocentric mental rotation. However, there were two limitations in the present study. As an exploratory study with a cross-sectional design, it was difficult to determine causality. Moreover, only one rotation axis, the front-back axis, was considered in our study, even though three different axes are often used by divers. To strengthen the present results, future studies need to add and manipulate the specific situations of the sport to the mental rotation task, such as movement information (e.g., rotation axes) or the sense of time to produce causal conclusions.

\section{ACKNOWLEDGEMENTS}

The authors thank the coaches of the diving team of Shanghai, Huiguo Shi and Xiaoling Yu, as well as the teacher of the students, Chao Li, for their approval of the experiment.

\section{REFERENCES}

Amorim, M.-A., Isableu, B., \& Jarraya, M. (2006). Embodied spatial transformations: "Body analogy" for the mental rotation of objects. Journal of Experimental Psychology: General, 135, 327-347. doi: 10.1037/0096-3445.135.3.327 |WW

Decety, J. (2002). Is there such a thing as functional equivalence between imagined, observed, and executed action? In A. N. Meltzoff \& W. Prinz (Eds.), The imitative mind: Development, evolution, and brain bases (pp. 291-310). New York, NY: Cambridge University Press.

Ericsson, K. A., Krampe, R. T., \& Tesch-Römer, C. (1993). The role of deliberate practice in the acquisition of expert performance. Psychological Review, 100, 363-406. doi: 10.1037/0033-295X.100.3.363

Habacha, H., Lejeune-Poutrain, L., Margas, N., \& Molinaro, C. (2014). Effects of the axis of rotation and primordially solicited limb of high level athletes in a mental rotation task. Human Movement Science, 37, 58-68. doi: 10.1016/j.humov.2014.06.002 [WW

Heil, M., \& Rolke, B. (2002). Toward a chronopsychophysiology of mental rotation. Psychophysiology, 39, 414-422. doi: 10.1017. S0048577202001105 WWW

Heinen, T. (2013). Does the athletes' body shape the athletes' mind? A few ideas on athletes' mental rotation performance. Commentary on Jansen and Lehmann. Advances in Cognitive Psychology, 9, 99-101. doi: 10.2478/v10053-008-0136-7 [WW]

Heinen, T., \& Jeraj, D. (2013). The athletes' body shapes the athletes' mind-new perspectives on mental rotation performance in athletes. Problems of Psychology in the 21st Century, 7, 23-31.

Heirani, A., Vazinitaher, A., Soori, Z., \& Rahmani, M. (2012). Relationship between choice reaction time and expertise in 
team and individual sports: A gender differences approach. Australian Journal of Basic \& Applied Sciences, 6, 344-348.

Heppe, H., Kohler, A., Fleddermann, M.-T., \& Zentgraf, K. (2016). The relationship between expertise in sports, visuospatial, and basic cognitive skills. Frontiers in Psychology, 7:904. doi: 10.3389/fpsyg.2016.00904 $\overline{\text { WWW }}$

Hoyek, N., Champely, S., Collet, C., Fargier, P., \& Guillot, A. (2014). Is mental rotation ability a predictor of success for motor performance? Journal of Cognition and Development, 15, 495-505. doi: 10.1080/15248372.2012.760158

lonta, S., \& Blanke, O. (2009). Differential influence of hands posture on mental rotation of hands and feet in left and right handers. Experimental Brain Research, 195, 207-217. doi: 10.1007/s00221-009-1770-0 WWW

Jansen, P., \& Heil, M. (2010). The relation between motor development and mental rotation ability in 5- to 6-year-old children. European Journal of Developmental Science, 4, 66-74. doi: 10.3233/DEV-2010-4105

Jansen, P., \& Kellner, J. (2015). The role of rotational hand movements and general motor ability in children's mental rotation performance. Frontiers in Psychology, 6:984. doi: 10.3389/ fpsyg.2015.00984 WWW

Jansen, P., Lange, L., \& Heil, M. (2011). The influence of juggling on mental rotation performance in children. Biomedical Human Kinetics, 3, 223-229. doi: 10.2478/v10101-011-0005-6 WWW

Jansen, P., \& Lehmann, J. (2013). Mental rotation performance in soccer players and gymnasts in an object-based mental rotation task. Advances in Cognitive Psychology, 9, 92-98. doi:

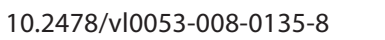

Jansen, P., Lehmann, J., \& Van Doren, J. (2012). Mental rotation performance in male soccer players. PLOS ONE, 7, 1310-1315. doi: 10.1371/journal.pone.0048620 $\underline{\underline{W W}}$

Jola, C., \& Mast, F. (2005). Mental object rotation and egocentric body transformation: Two dissociable processes? Spatial Cognition \& Computation, 5, 217-237. doi: 10.1207/ s15427633scc052\&3_6

Just, M. A., \& Carpenter, P. A. (1985). Cognitive coordinate systems: Accounts of mental rotation and individual differences in spatial ability. Psychological Review, 92, 137-172. doi: 10.1037/0033-295X.92.2.137 WWW

Kaltner, S., \& Jansen, P. (2015). Does body awareness influence visual spatial intelligence? International Journal of Learning, Teaching and Educational Research, 13, 1-13. doi: 10.1037/0033295X.92.2.137

Kaltner, S., Riecke, B. E., \& Jansen, P. (2014). Embodied mental rotation: A special link between egocentric transformation and the bodily self. Frontiers in Psychology, 5:505. doi: 10.3389/ fpsyg.2014.00505 wwW

Kioumourtzoglou, E., Kourtessis, T., Michalopoulou, M., \& Derri, V. (1998). Differences in several perceptual abilities between experts and novices in basketball, volleyball and water- polo. Perceptual \& Motor Skills, 86, 899-912. doi: 10.2466/ pms.1998.86.3.899 $\overline{\mathrm{WWW}}$

Liang, Y. M. (2014). The information procession and neural mechanism of diving motor imagery and temporal and spatial perception. Shanghai University of Sport.

Moreau, D. (2015). Unreflective actions? Complex motor skill acquisition to enhance spatial cognition. Phenomenology and the Cognitive Sciences, 14, 349-359. doi: 10.1007/s11097-0149376-9

Moreau, D., Clerc, J., Mansy-Dannay, A., \& Guerrien, A. (2011). Spatial ability and motor performance: Assessing mental rotation processes in élite and novice athletes. International Journal of Sport Psychology, 54, 167-179.

Moreau, D., Clerc, J., Mansy-Dannay, A., \& Guerrien, A. (2012). Enhancing spatial ability through sport practice. Journal of Individual Differences, 33, 83-88. doi: 10.1027/1614-0001/ a000075

Ozel, S., Larue, J., \& Molinaro, C. (2002). Relation between sport activity and mental rotation: Comparison of three groups of subjects. Perceptual \& Motor Skills, 95, 1141-1154. doi: 10.2466/

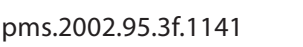

Ozel, S., Larue, J., \& Molinaro, C. (2004). Relation between sport and spatial imagery: Comparison of three groups of participants. The Journal of Psychology, 138, 49-64. doi: 10.3200/ JRLP.138.1.49-64 WWW

Pietsch, S., \& Jansen, P. (2012). Different mental rotation performance in students of music, sport and education. Learning and Individual Differences, 22, 159-163. doi: 10.1016/j. lindif.2011.11.012

Piras, A., Lobietti, R., \& Squatrito, S. (2014). Response time, visual search strategy, and anticipatory skills in volleyball players. Journal of Ophthalmology, 2014, 1-10. doi: $10.1155 / 2014 / 189268$ |WWW

Reed, C. L. (2002). Chronometric comparisons of imagery to action: Visualizing versus physically performing springboard dives. Memory \& Cognition, 30, 1169-1178. |WwW

Schmidt, M., Egger, F., Kieliger, M., Rubeli, B., \& Schüler, J. (2015). Gymnasts and orienteers display better mental rotation performance than non-athletes. Journal of Individual Differences, 37, 1-7. doi: 10.1027/1614-0001/a000180

Shepard, R. N., \& Cooper, L. A. (1986). Mental images and their transformations. Cambridge, MA: MIT Press.

Shepard, R. N., \& Metzler, J. (1971, February). Mental rotation of three-dimensional objects. Science, 171(3972), 701-703. doi: 10.1126/science.171.3972.701 www

Steggemann, Y., Engbert, K., \&Weigelt, M. (2011). Selective effects of motor expertise in mental body rotation tasks: Comparing object-based and perspective transformations. Brain \& Cognition, 76, 97-105. doi: 10.1016/j.bandc.2011.02.013 WWW

Vereza, S. C. (1999). Philosophy in the flesh: The embodied mind and its challenge to Western thought. New York, NY: Basic Books. 
Voyer, D., \& Bryden, M. (1990). Gender, level of spatial ability, and lateralization of mental rotation. Brain \& Cognition, 13, 18-29. doi: 10.1016/0278-2626(90)90037-O WWw

Voyer, D., \& Jansen, P. (2017). Motor expertise and performance in spatial tasks: A meta-analysis. Human Movement Science, 54, 110-124. doi: 10.1016/j.humov.2017.04.004 WWW

Wexler, M., Kosslyn, S. M., \& Berthoz, A. (1998). Motor processes in mental rotation. Cognition, 68, 77-94. doi: 10.1016/S00100277(98)00032-8 WWW

Wilson, M. (2002). Six views of embodied cognition. Psychonomic Bulletin \& Review, 9, 625-636. doi: 10.3758/BF03196322 WWW

Wohlschläger, A., \& Wohlschläger, A. (1998). Mental and manual rotation. Journal of Experimental Psychology: Human Perception and Performance, 24, 397-412. doi: 10.1037/0096-1523 .24.2.397 $\underline{\underline{W W}}$
Wright, R., Thompson, W. L., Ganis, G., Newcombe, N. S., \& Kosslyn, S. M. (2008). Training generalized spatial skills. Psychonomic Bulletin \& Review, 15, 763-771. doi: 10.3758/PBR.15.4.763 |wWW Yu, A. B., \& Zacks, J. M. (2015). How are bodies special? Effects of body features on spatial reasoning. Quarterly Journal of Experimental Psychology, 69, 1210-1226. doi: 10.1080/17470218.2015.1079225 WWW

Zacks, J. M., Ollinger, J. M., Sheridan, M. A., \& Tversky, B. (2002). A parametric study of mental spatial transformations of bodies. Neuroimage, 16, 857-872. doi: 10.1006/nimg.2002.1129 WWW Zwierko, T., Osinski, W., Lubinski, W., Czepita, D., \& Florkiewicz, B. (2010). Speed of visual sensorimotor processes and conductivity of visual pathway in volleyball players. Journal of Human Kinetics, 23, 21-27. doi: 10.2478/v10078-010-0003-8

RECEIVED 17.04.2017 | ACCEPTED 01.08.2017 